\title{
Identity Checking System by Light Blinking ID-Beacon Using Image Sensor
}

\author{
Boonchana Purahong ${ }^{+}$ \\ Faculty of Engineering, King Mongkut's Institute of Technology Ladkrabang, Bangkok, Thailand
}

\begin{abstract}
In this paper, a new method for the ID checking system was proposed by using the Visible Light Communication (VLC). The proposed method uses an image sensor of webcam camera and smartphone that can use fulfill some limitations of QRCode and RFID technology. In the experiment, ID the system can identify identity codes transmitted in 1-50 meters in daytime and night to find the processing time for the first time to detect LED Blinking. As the result, the performance of ID checking can use webcam camera to identify objects the maximum distance in daytime and night but smartphone can identify in short distance at night. Comparing a performance for application in various systems between webcam camera and smartphone image sensor.
\end{abstract}

Keywords: light blinking, ID Beacon, VLC.

\section{Introduction}

The ID checking system is decisive for real-life security system. Although today identification technologies are relied on QRcode and RFID technologies, but both of the systems has its some limitation on distance, cost night vision, and regulation on frequency. Identity checking system by visible light [1] is recent work for application to identification systems using visible light communication.

In the past, There have been some related system for ID Checking. For example, used infared LED in museum [2], ID Recognizing systems with IR-LEDs application with balloon tags. [3] and some related systems used smartphone image sensor to detect a low-frame rate of color transforming on the smartphone screen. [4] And some related system used smart image sensor for indoor positioning system [5]. Visible light communication with light signaling is a method to transmit data from light emitting. There are several advantages of this communication method compared to other wireless communication methods including radio waves. The visible light is not harmful to human body and it is able to be transmitted with high power. Moreover, the visible light is secure to use and line of sight. Illuminate from LED not only illuminate but also use to send data by different blinking pattern may be blinking in high frequency and human cannot see how to blink. Receiver can use photodiode or image sensor but, this system used webcam camera and smartphone camera (image sensor) because image sensor can receive multiple signals, robustness and long distance more than photodiode.

In this paper, the Identity checking system was designed to be a portability, suitability for application various systems be used a webcam camera and smartphone image sensor in receiver method with visible light communication and compare a performance with webcam camera image sensor to find a suitable application.

\section{System Overview and Design}

\footnotetext{
+ Corresponding author. Tel.: +6681403 3711

E-mail address:_mr.neng49@gmail.com
} 
Our system is composed of 3 main points, transmitter, receiver and database management. Transmitter has a microcontroller for transmitting data by LED blinking ID-beacon. That one blinking pattern instead of one identity of object by bit 1 is on and bit 0 is off and get a code blinking pattern of object to save in database. Receiver using an image sensor to use for detecting LED blinking ID-beacon. The overview of this system shows in Fig. 1

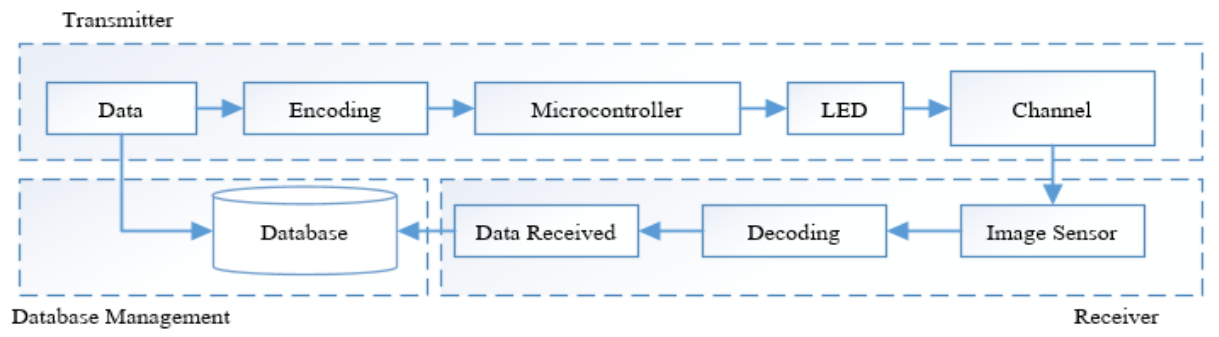

Fig. 1: System overview.

\subsection{Transmitter}

In Transmitter method using a microcontroller to control LED blinking ID-beacon. This paper use a adruino nano to control LED blinking ID-beacon for transmitting data that show in Fig. 2.

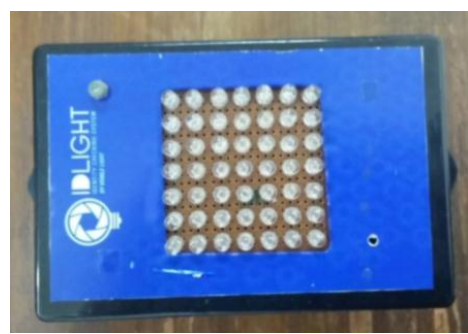

Fig. 2: LED beacon controlled by Arduino Nano.

For controlling a blinking of LED ID-beacon, the system define a blinking pattern into a decimal number of identity code for one of data. Identity code is a primary key of database. After that, convert a decimal identity code to binary code for encryption. The sequence of binary code divided into three parts, consisting of header bit, data bit and close bit and transmit in two patterns, 26 bit for webcam camera and 16 bit for smartphone camera due to a limitation of frame rate.

For the encoding, our system was defined the sequence of binary bits and encrypt that bit 1 is LED on and bit 0 is LED off. And then programming the identity code into a microcontroller for blinking a light of LED ID-beacon. The overview of transmitter method that shows in Fig. 3.

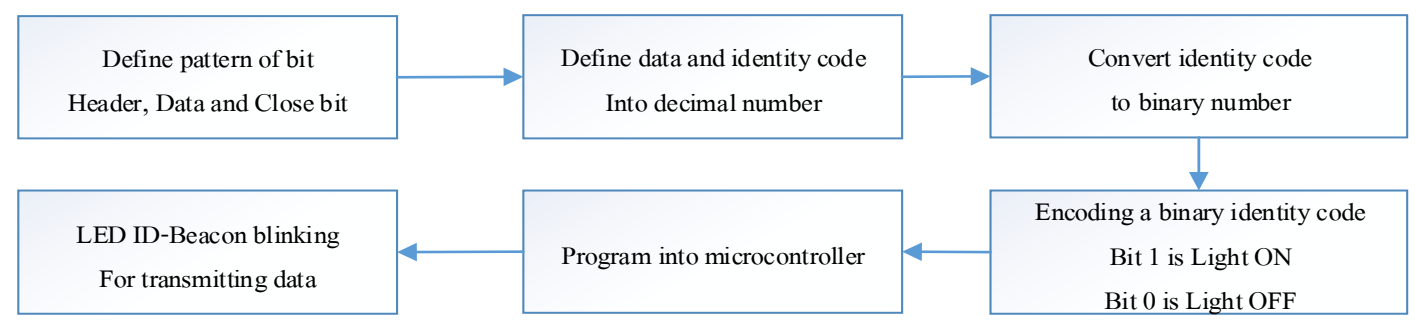

Fig. 3: Overview of transmitter method.

\subsection{Receiver}

In the receiver method, for detecting and analyzing a pattern of LED blinking ID-beacon, Our system used an image sensor of webcam camera and smartphone camera for comparing a performance of each type of image sensor in order to find the suitable application for each camera. A Webcam camera in this system using OKER-177 webcam camera that is inexpensive and good performance webcam camera and use frame rate of 25 30 fps. A Smartphone camera are used Samsung Galaxy Note II with android operating system to 
identify the light blinking. The system using webcam camera was developed by Visual-C\# compatible with OpenCV, EmguCV and AForge.NET library that are image processing libraries. The system using smartphone camera was developed by JAVA and android studio compatible with OpenCV library. The interface of the system both C\# and smartphone application and display when detect a light blinking.

The identity checking system stores identity of data or objects in form of identity code. As to present an identity, each code of the data or object's identity is encoded and converted to be a sequence of LED blinks controlled by a microcontroller within the transmitter method. The receiver software perceives the blinking pattern by determining a series of frame that contain different level of LED luminance. Luminance of an image could be calculated from Y channel of YUV codes converted from RGB. And then the receiver software or application is already received a series of luminance ( $\mathrm{Y}$ channel) frames. After that, software is calculated the threshold or average of luminance ( $Y$ channel) frame. This method is explained by (1). Threshold means an average of Y channel frame and Y channel each frame Yi and total frame fp.

$$
\text { Threshold }=\frac{\sum_{i=1}^{f p} Y i}{f p}
$$

The luminance level is compared with a defined threshold to be classified as LED ON(bright) " 1 " or LED OFF(dark) " 0 ". The decoded code would be used to search through a database to find a matched one. In (2) And Fig.4 shows algorithm to analyze blinking pattern.

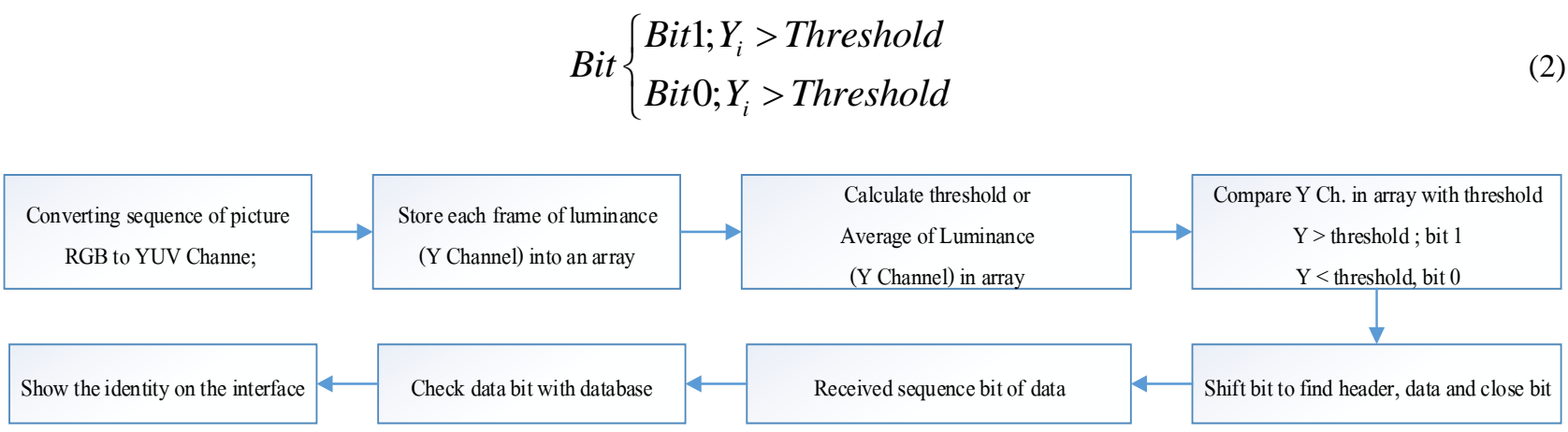

Fig. 4: Algorithm to analyze the blinking pattern.

\section{Experimental Results}

The results of this system was experimented in daytime and night at distance between transmitter and receiver 1 to 50 meters with a little different of environment using webcam camera with 26-bit transmitting and smartphone camera application with 16-bit transmitting because smartphone camera can detect in low frame rate.

The example of this system when the LED ID-Beacon blinking are detected by smartphone image sensor and The example setting of light blinking ID-beacon in daytime and night show in Fig. 5 After that, the application display identity of objects when received a correct data. And the results of experiment that show in Table 1.

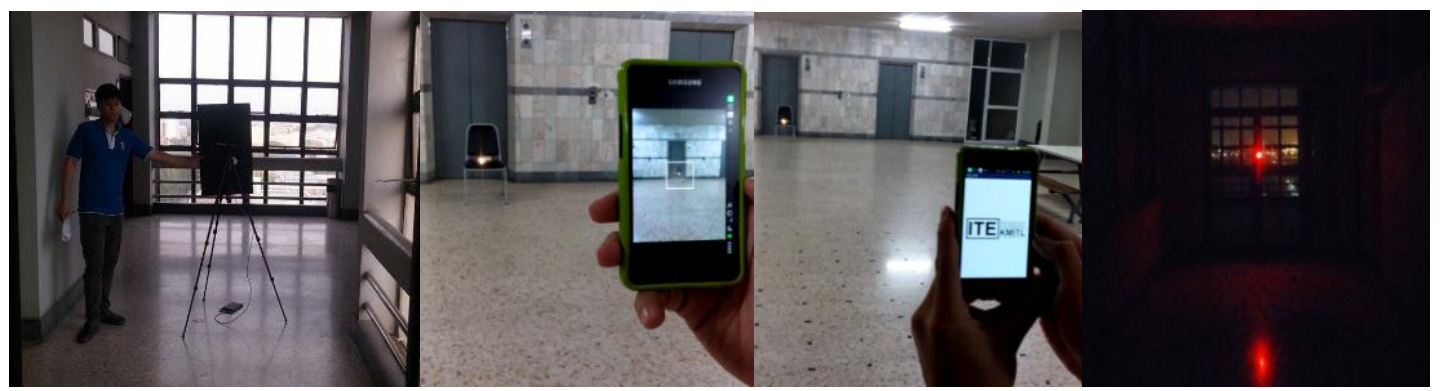

Fig. 5: Example of smartphone. 
Table 1: Experimental Results

\begin{tabular}{|c|c|c|c|c|}
\hline \multirow{3}{*}{$\begin{array}{l}\text { Distance } \\
\text { (Meters) }\end{array}$} & \multicolumn{4}{|c|}{ Average of the first time to detect a LED blinking ID-beacon (Second) } \\
\hline & \multicolumn{2}{|r|}{ Daytime } & \multicolumn{2}{|c|}{ Night } \\
\hline & Webcam & Smartphone & Webcam & Smartphone \\
\hline 1 & 0.75 & 2.13 & 0.76 & 2.02 \\
\hline 5 & 0.83 & 2.23 & 0.77 & 2.43 \\
\hline 10 & 0.84 & 3.12 & 0.78 & 2.23 \\
\hline 20 & 1.31 & 3.32 & 0.81 & 2.33 \\
\hline 30 & 2.54 & 4.45 & 0.86 & 3.19 \\
\hline 40 & 4.17 & - & 0.83 & 3.36 \\
\hline 50 & - & - & 0.85 & 3.97 \\
\hline
\end{tabular}

From Table 1, In daytime, When used a webcam camera to detect light blinking ID-beacon. It have a performance for detecting in distance 1 meter to 40 meter with 0.75 second to 4.17 second. When used a smartphone camera to detect light blinking ID-beacon. It have a performance for detecting in distance 1 to 30 meter. At night, When used a webcam camera to detect. It have a good performance for detecting along 1 to 50 meter distance with 0.76-0.86 second. And Smartphone camera have a performance for detecting in distance 1 to 50 meter with $2.02-3.97$ second. The results show the webcam camera maybe good performance more than smartphone camera. Actually, both system have an various of advantage and disadvantage that show a summary in Table 3 .

Table 3: Summary of identity checking system using image sensor

\begin{tabular}{|c|c|c|}
\hline Topics & Webcam & Smartphone \\
\hline Frame rate & $25-30 \mathrm{fps}$ & $10-15 \mathrm{fps}$ \\
\hline Cost & - Additional cost & - No additional cost \\
\hline Performance & $\begin{array}{l}\text { - High frame rate and it can store more identity code } \\
\text { or data. } \\
\text { - Detection farther and faster both daytime and night }\end{array}$ & $\begin{array}{l}\text { - Low frame rate and storage of identity code is no more. } \\
\text { - Lower performance and maybe depend on type of smartphone image } \\
\text { sensor. }\end{array}$ \\
\hline Comfortable & $\begin{array}{l}\text { - Uncomfortable and not portable for using. } \\
\text { - It always connect webcam with computer }\end{array}$ & - Comfortable and portable almost people use a smartphone generally. \\
\hline Application & $\begin{array}{l}\text { - Suitable for close system that limit access to } \\
\text { specific people or the system needed to detect at a } \\
\text { far distance such as specific identification system, } \\
\text { vehicle communication }\end{array}$ & $\begin{array}{l}\text { - Suitable for open system that unlimited for people access and easy to use } \\
\text { by smartphone with short distance, indoor detecting or low light. For } \\
\text { example, an advertisement system, museum system, indoor positioning } \\
\text { system. }\end{array}$ \\
\hline
\end{tabular}

\section{References}

[1] Boonchana Purahong, Thanavit Anuwongpinit and Jirattawut Dumrong "Identity Checking System by Visible Light" The 4th Join International Conference on Information and Communication Technology, Electronic and Electrical Engineering (JICTEE2014), March 5-8, 2014, Chiang Rai, Thailand.

[2] Jun Park, Kyu Hwa Lee, Sang Goo Lee, “ID Recognition for Museum Tourists Using Infared LED's " Second Workshop on Digital Media and its Application in Museum \& Heritage p.115-117

[3] H.Aoki, D.Mstsushita., "Balloon Tag (In)visible Marker Which Tells Who's Who", The Fourth International Symposium on Wearable Computers2000,pp.181-182

[4] Picapi Camera : http://www.casio-isc.com/en/help/

[5] Pang G.K.H.,Liu H.H.S., "LED location beacon system based on processing of digital images", Intelligent Transportation Systems, IEEE Transactions on Volume:2, Issue: 3, August, 7, 2003, pp.135 - 150. 